AperTO - Archivio Istituzionale Open Access dell'Università di Torino

Metaflammation: tissue-specific alterations of the NLRP3 inflammasome platform

This is a pre print version of the following article:

Original Citation:

Availability:

This version is available http://hdl.handle.net/2318/1634181

since 2018-07-16T11:44:24Z

Published version:

DOI:10.2174/0929867324666170407123522

Terms of use:

Open Access

Anyone can freely access the full text of works made available as "Open Access". Works made available under a Creative Commons license can be used according to the terms and conditions of said license. Use of all other works requires consent of the right holder (author or publisher) if not exempted from copyright protection by the applicable law. 


\title{
Metaflammation: tissue-specific alterations of the NLRP3 inflammasome platform in metabolic syndrome \\ Raffaella Mastrocola ${ }^{\mathrm{a}}$, Manuela Aragno ${ }^{\mathrm{a}}$, Giuseppe Alloatti ${ }^{\mathrm{b}}$, Massimo Collino $^{\mathrm{c} *}$, \\ Claudia Penna ${ }^{\mathrm{a}}$, Pasquale Pagliaro ${ }^{\mathrm{a}}$.
}

\begin{abstract}
Affiliations:
${ }^{a}$ Department of Clinical and Biological Sciences, University of Turin, Torino, Italy; ${ }^{b}$ Department of Life Sciences and Systems Biology, University of Turin, Torino, Italy; 'Department of Drug Science and Technology, University of Turin, Torino, Italy.
\end{abstract}

\section{*Corresponding author:}

Massimo Collino,

Department of Drug Science and Technology,

University of Turin,

via P. Giuria 9, 10125 Turin, Italy.

Tel. 00390116706861

E-mail: massimo.collino@unito.it

\section{Review sections:}

1. INTRODUCTION

2. METABOLIC SYNDROME AND INFLAMMATION: METAFLAMMATION

3. THE NLRP3 INFLAMMASOME PLATFORM IN METABOLIC SYNDROME

3.1 NPLR3: mechanisms of activation

3.2 ROS and AGEs as NLRP3 inducers in metabolic syndrome

4. NLRP3 IN TARGET TISSUES OF METAFLAMMATION

4.1. Heart: NLRP3 inflammasome in myocardial ischemia/reperfusion, an overview

4.2. Liver: NLRP3 inflammasome in steatohepatitis, an overview

4.3. Kidney: NLRP3 inflammasome in diabetic nephropathy, an overview

5. THERAPEUTIC INTERVENTIONS TARGETING NLRP3 ACTIVATION IN METAFLAMMATION

6. CONCLUSIONS 


\begin{abstract}
In the last decades, the extension of life expectancy and the increased consumption of foods rich in saturated fats and added sugars have exposed the general population to emerging health problems.

The prevalence of metabolic syndrome (MS), composed of a cluster of factors as obesity, dyslipidemia, hyperglycemia, and hypertension, is rapidly increasing in industrialized and developing countries leading to precocious onset of age-related diseases. Indeed, oxidative stress, accumulation of advanced glycation endproducts, and a chronic low-grade inflammation are common features of MS and physiological ageing. In particular, the entire set of MS factors contributes to the development of an inflammatory status named metaflammation, which has been associated with activation of early innate immune response through the assembling of the multiprotein complex inflammasome. The most investigated family of inflammasome platforms is the NOD-like receptor pyridine containing (NLRP) 3 , which is activated by several exogenous and endogenous stimuli, leading to the sequential cleavage of caspase-1 and IL-1 $\beta$, followed by secretion of active IL-1 $\beta$. We here collect the most recent findings on NLRP3 activation in MS providing evidence of its central role in disease progression and organ dysfunction in target tissues of metaflammation, in particular in cardiovascular, hepatic and renal complications, with a focus on oxidative stress and advanced glycation endproducts. A wide overview of the most promising strategies for the modulation of NLRP3 activation and related metabolic repercussions is also provided, since the finding of specific pharmacological tools is an urgent requirement to reduce the social and economic burden of MS- and elderly-associated diseases.
\end{abstract}

Keywords: metabolic syndrome; metaflammation; NLRP3 inflammasome; oxidative stress; aging; myocardial ischemia/reperfusion; steatohepatitis; chronic kidney disease. 


\section{INTRODUCTION}

In the last decades, two fundamental changes in the modern society have occurred: extension of lifespan and modification of eating habits. Even if improvements in public health care and advances in medical science have significantly extended life expectancy, on the other side the increased consumption of industrialized foods, high in saturated fats and added sugars, has exposed the general population to emerging health problems [1,2]. The onset of metabolic disturbances accompanying ageing, as obesity, metabolic syndrome (MS), and diabetes, has reached global epidemic proportions. Indeed, the World Health Organization has reported that worldwide 400 million adults are obese, about $6 \%$ of the general population. Particularly in the western countries the rate of obesity within the aging population is dramatically increasing: in the United States in the period between 2007 and 2010 about 8 million of adults aged 6574 were obese and it is expected that obese individuals will be at least the $75 \%$ of population by 2025 [3].

Frequently, obesity is accompanied by a cluster of other factors, as dyslipidemia, hyperglycemia and hypertension, that taken together define the MS and increase the risk for the occurrence of diabetes and cardiovascular diseases (CVD), as well as hepatic and renal dysfunction [4-7].

Indeed, many components of the MS, as obesity, hyperglycemia and hyperlipidemia, are individually associated to the establishment of a chronic low grade inflammation and oxidative stress that contribute to the development of the above-mentioned MS-related diseases [1,8]. Interestingly, a decline in innate immune response that generates a permanent inflammatory status and the accumulation of free radicals and glicoxidation and lipoperoxidation compounds, as advanced glycation endproducts (AGEs), are common features of the physiological ageing [9-12]. Thus, MS can be considered a condition that accelerates the natural mechanisms of ageing and lead to the precocious development of organ dysfunction [13].

This severely affects the social and economic burden of elderly-associated diseases, and MS should be considered as an important pathological process that requires to be specifically addressed. It is, therefore, mandatory to develop responsible strategies aimed at investigating and dissecting the mechanisms underlying MS in order to foresee therapeutic approaches.

\section{METABOLIC SYNDROME AND INFLAMMATION: METAFLAMMATION}

There is clearly increased risk of MS in obesity, which is also a major risk factor for a number of chronic diseases, including cancer, stroke, cardiovascular complications and premature death. In particular, there is strong evidence for the causative association between central (visceral) obesity and MS and the development of type 2 diabetes and cardiovascular complications, though the mechanisms are not fully understood [14]. Thus, the term metaflammation has been coined to define a low-grade, chronic inflammation orchestrated by metabolic cells in response to excess nutrients and energy in metabolic tissues including adipose, liver, muscle, pancreas, and brain [15].

The high level of coordination of inflammatory and metabolic pathways is highlighted by the overlapping biology and function of macrophages and adipocytes in obesity. Pre-adipocytes may exhibit anti-microbial and phagocytic properties and may differentiate into macrophages in the appropriate environment. Therefore, a potential immune role for pre-adipocytes has been suggested [16]. Furthermore, in obesity, macrophages and adipocytes co-localize in white adipose tissue. In this tissue, macrophages and/or adipocytes can contribute to the production of inflammatory mediators; thus macrophages may exert a potentially important influence in promoting insulin resistance. Besides macrophages, obesity is associated with aberrant expansion of other leukocytes ( $T$ cells, B cells, eosinophils, neutrophils, mast cells, and dendritic cells) in adipose tissue that contribute to chronic inflammation. In particular, the increased neutrophils lead to a rise of myeloperoxidase activity, a marker of neutrophil infiltration, in the damaged 
tissue during inflammation [17]. Moreover, the recruitment of circulating dendritic cells through the release of adipokines from adipose tissue in response to systemic free fatty acids increase has been previously demonstrated [18]. Specifically, infiltrating dendritic cells in adipose tissue are activated by the interaction between HMGB1 bound to the DNA molecules and RAGE receptor on dendritic cells and thus participate in the initiation of metaflammation through the release of interferon-gamma [18].

Despite the fact that specific inflammatory pathways that mediate the link between MS and the majority of chronic diseases have not yet been identified, most recent literature evidences suggest metaflammation to be a crucial link between the development of metabolic disorders and increased risk of CVD. In fact, visceral obesity and related insulin resistance increase cardiovascular risk by classical mechanisms such as dyslipidemia, hypertension and glucose dysmetabolism, and less conventional mechanisms, involving metaflammation [19]. Among less conventional risk factors there are adipokines, such as leptin and adiponectin, and proinflammatory cytokines, as interleukin(IL)-6 and tumor necrosis factor (TNF)- $\alpha$, which are secreted by adipocytes and macrophages infiltrating adipose tissue that is considered an active endocrine organ. Altogether, these factors not only affect body weight homeostasis, but might lead to increased oxidative stress, endothelial dysfunction and vascular inflammation [20]. This process leading to monocyte recruitment, foam cell formation and subsequent development of fatty streaks, over many years, promotes atherosclerotic plaques, which, in the presence of enhanced inflammatory conditions, may become unstable and undergo to rupture; thus promoting occlusive thrombus formation [21].

\section{THE NLRP3 INFLAMMASOME PLATFORM IN METABOLIC SYNDROME}

Before to start with considerations on MS and NOD-like receptor pyiridin domain containing (NLRP) 3, a brief general description of inflammasome, and in particular NLRP3 inflammasome, is necessary.

The term inflammasome was proposed in 2002 by Martinon and collaborators to describe a high-molecularweight complex present in the cytosol of stimulated immune cells that determines the activation of inflammatory caspases [22]. Danger signals can be sensed by the cells through a group of pattern recognition receptors (PRRs) able to recognize structures conserved among microbial species, which are referred to as pathogen-associated molecular patterns (PAMPs), or as endogenous molecules released from damaged cells, called damage-associated molecular patterns (DAMPs). The PRRs are expressed not only in immune cells, particularly macrophages, but also in various non-immune cells. To date, four different classes of PRR families have been described. These families include transmembrane and cytoplasmic proteins. While the former includes C-type lectin receptors (CLRs) and the Toll-like receptors (TLRs), the latter comprise the Retinoic acid-inducible gene (RIG)-I-like receptors (RLRs) and NOD-like receptors (NLRs) [23]. Among the NLRs, the best-characterized is the NLRP3 inflammasome (also known as NALP3 and cryopyrin), which interacts with an apoptosis-associated speck-like protein including a caspase recruitment domain (ASC), thus recruiting and activating caspase-1. Since caspase-1 is an IL-1ß-converting enzyme, it mediates the transformation of pro-IL-1 $\beta$ into mature IL-1 $\beta$, which is released causing inflammation. Yet, the transcriptional induction of pro-IL- $1 \beta$ is required to have the induction of IL- $1 \beta$ release. Thus, a system including pro-IL- $1 \beta$ induction and inflammasome-mediated IL-1 $\beta$ maturation seems necessary for the regulation of this inflammatory cytokine [24].

Most recent evidence has demonstrated the emerging role as a unifying cause of disease of the NLRP3inflammasome in DAMPs-induced ageing-related pathologies [25]. Inadequate inflammasome activity has been indeed involved in the pathogenesis of inflammatory diseases and metabolic disorders, and, interestingly, in ageingrelated diseases pathology-specific ligands (such as cholesterol crystals, oxidation-specific epitopes for atherosclerosis; A $\beta$ for Alzheimer Disease; $\alpha$-synuclein for Parkinson's disease; drusen, tiny clear accumulations of extracellular 
material in the retinal pigment epithelium of the eye, for age-related macular degeneration and monosodium urate for gout) drive sterile inflammation through the activation of inflammasome [26-29].

So far, no specific DAMPs have been identified linking ageing to MS, though we and others have recently contributed to demonstrate that NLRP3 inflammasome plays a pivotal role in the induction of insulin resistance and cardiac ischemia/reperfusion injury exacerbation [30-34]. Besides insulin resistance, it has also been proposed that NLRP3 "senses" obesity-associated danger signals and may contribute to obesity-induced inflammation [35].

Given that MS is strictly related with increased risk for myocardial infarct and worse ischemia/reperfusion outcomes, we here review recent findings on the role of NLRP3 in different target organs of metabolic disturbances, with a focus on cardiac ischemia/reperfusion injury, hepatosteatosis, and diabetic nephropathy.

\subsection{NLPR3: mechanisms of activation}

The NLRP3 inflammasome is activated in response to a large number of stimuli, including the changes in intracellular calcium levels, the production of reactive oxygen species (ROS) and the release of oxidized mitochondrial DNA, as well as in response to mitochondrial dysfunction, lysosomal destabilization, the formation of large nonspecific plasma-membrane pores and potassium efflux.

However, the exact mechanisms of NLRP3 activation are not completely understood. It seems that NLRP3 is activated by a diverse range of agonists that differ both structurally and chemically with an unclear activation mechanism [36]. Actually, NLRP3 does not bind directly with any of its putative agonists/activators. In particular, no studies have yet demonstrated an interaction between the sensing domain of NLRP (LRR, leucine rich region) and any NLRP3 agonists. At moment, four different modalities of NLRP3 activation have been suggested: 1) mitochondrial ROS generation, 2) phagolysosomal destabilization, 3) potassium efflux, and 4) osmotic pressure and cell volume regulation.

1) Several NLRP3 activators induce ROS formation from damaged mitochondria, which may activate the NLRP3 via an intermediate, such as the released mitochondrial DNA or the thioredoxin-interacting protein (TNXIP), which binds to NLRP3, thus obtaining its activation [37]. A recent study has proposed that ROS are necessary for priming the NLRP3 inflammasome, but not for its activation [38].

2) Large particulate matter, including alum, amyloid- $\beta$, and silica, may activate the inflammasome. Intracellular clogging of these particles leads to the formation and subsequent swelling of phagolysosome. Therefore, lysosomal matter, including cathepsin B is released and sensed by NLRP3 inflammasome, leading to its assembly [39].

3) Intracellular $\mathrm{K}^{+}$efflux, obtained via the activation of purinergic receptor 7 (P2X7)-ATP-gated ion channels by extracellular ATP, results in NLRP3 inflammasome oligomerization. ATP-induced activation of P2X7 receptors has also been linked to pannexin-1 dependent formation of large nonselective cell membrane pores, which would allow NLRP3 activators to enter into the cells and to activate directly or indirectly NLRP3 [40,41].

4) Finally, osmotic pressure and cell volume regulation have been associated to NLRP3 activation. It seems that hypotonic solution triggers a decrease in intracellular $\mathrm{K}^{+}$and $\mathrm{Cl}^{-}$concentrations and a regulatory response leading to cellular volume decrease. This is due to the intervention of transient receptor potential (TRP) and transient receptor potential cation channel subfamily M member 7 (TRPM7), which mediate the intracellular $\mathrm{Ca}^{2+}$ handling. Both intracellular $\left[\mathrm{Ca}^{2+}\right]$ variation and TRP channel-dependent volume decrease response may lead to the formation of caspase-1 [42]. This is in line with the finding that intracellular $\mathrm{Ca}^{2+}$ may activate the transforming growth factor $\beta$-activated kinase 1(TAK1) and that this enzyme plays a role in NLRP3 activation [43]. 
Since a unified mechanism for NLRP3 activation lacks, it has been put forward the idea that different agonists may evoke similar downstream events that are sensed by NLRP3 [44]. Actually, the inflammasome products, IL-1 $\beta$ and IL-18, are targeted by many reagents, including the neutralizing IL-1 $\beta$ antibody canakinumab, the recombinant IL-1RA anakinra, the soluble decoy IL-1 receptor rilonacept, the IL-18-binding protein, the soluble IL-18 receptors and, indeed, the anti-IL-18 receptor monoclonal antibodies have been developed in the attempt to treat autoinflammatory diseases. However, only the evaluation of small molecules able to selectively inhibit the NLRP3 inflammasome can allow a better understanding of the role of the NLRP3 inflammasome-caspase-1-IL-1ß/18 axis in the progression of specific diseases and may allow the future design of effective therapeutics. Notably, selective inhibition of NLRP3 by small molecules might have advantages compared with biological agents targeted at IL-1 $\beta$ and its receptor, including fewer immunosuppressive effects and better pharmacokinetics and cost-effectiveness. Unfortunately, so far, there has not been an effective development of inflammasome NLRP3 inhibitors. We recently tested, in vitro and in ex vivo model a small molecule as promising selective inhibition of NLRP3 [45].

\subsection{The ROS and AGEs as NLRP3 inducers in metabolic syndrome}

Clearly, the deterioration of the structure and function of organs during ageing is associated with oxidative stress, and disruption of homeostatic pathways. Lifelong exposure to different stressors results in a chronic oxidative stress process, leading to oxidative damage in biomolecules, a process thought to be a strong contributor to the process of ageing [46]. This oxidative situation leads to the release of DAMPs that initiate a sterile inflammation process [47].

ROS may also result in the formation of AGEs, which are the product of the non-enzymatic reaction of reducing carbohydrates with lysine side chains and $\mathrm{N}$-terminal amino groups of macromolecules (proteins, phospholipids and nucleic acids), that is the so-called Maillard reaction or glycation. Accumulation of AGEs has been evidenced in several target tissues of MS and as consequence of excessive intake of saturated fats and sugars [48-52]. Importantly, the progressive decline in mitochondrial efficiency during ageing is accompanied by increasing ROS levels and paralleled by accumulating AGEs in tissues [53]. In this context, a lifetime consumption of a high-AGEs-generating diet may evoke an acceleration of ageing processes [54]. AGEs, adversely affect the functional properties of proteins, lipids and DNA, and an association between augmented accumulation of AGEs and their receptor RAGE and an increased expression and activation of NLRP3 inflammasome has been hypothesized [55,56]. Actually, this association has been recently reported in pancreatic islets of obese and galectin-3, another receptor for AGEs, ablated animals. These effects were also associated with elevated phosphorylation of nuclear factor- $\mathrm{kB}$ (NF-kB), p65 and mature caspase-1 protein expression in both pancreatic and visceral adipose tissues [57]. Whether AGEs can activate NLRP3 directly acting as DAMPs or by the binding to AGEs-receptors remains to be ascertained. In particular, the complex redox-dependent pathway from AGEs to NLRP3 may include the inhibition of AMP-activated protein kinase (AMPK), thus exerting a control over mitochondrial metabolism, and therefore on inflammatory status of many cell types [58]

Understanding how AMPK-dependent pathways and the related pro-survival Reperfusion Injury Salvage Kinases (RISK) and Survivor Activating Factor Enhancement (SAFE) pathways regulate oxidative metabolism and substrate selection to control NLRP3 inflammasome-mediated inflammation holds promise for identifying new therapies for the treatment of MS dependent pathologies. Actually, in a recent article we have shown that diet-induced exacerbation of myocardial ischemia/reperfusion (I/R) injury evokes upregulation of NLRP3, which is associated to a downregulation of cardioprotective pathways, including RISK [33]. Further studies might clarify whether an inhibition of AGEs accumulation or AGEs-receptors signaling may restore protective pathways through the limitation of NLRP3 activation. 


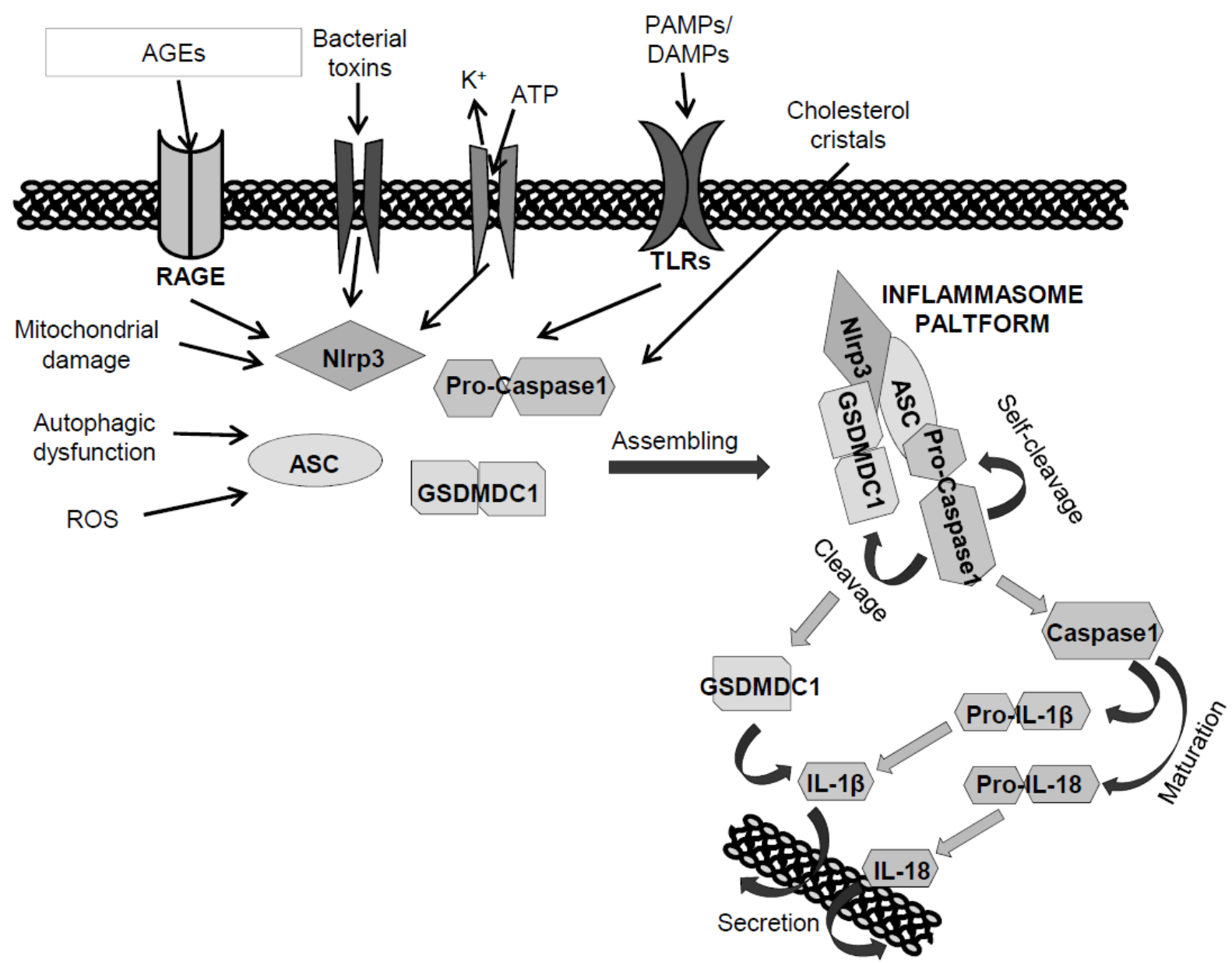

Figure 1. Schematic illustration of molecular/signalling pathways that activate and are being activated by NLPR3. Several danger signals, as well as advanced glycation end products (AGEs) and reactive oxygen species (ROS), elicit NLRP3 to recruit the cytoplasmic components to assemble inflammasome platform. After assembling, pro-caspase-1 undergoes a selfcleavage into active caspase-1, which in turn activates gasdermin (GSDMDC1) cleavage, and pro-IL-1 $\beta$ and pro-IL18 maturation. Finally, the activated GSDMDC1 promotes active IL-1 $\beta$ and IL-18 secretion.

\section{NLRP3 IN TARGET TISSUES OF METAFLAMMATION}

\subsection{Heart: NLRP3 inflammasome in myocardial ischemia/reperfusion, an overview}

As previously mentioned, MS-related metaflammation boosts the risk of CVD and correlated comorbidities, such as type 2 diabetes. The correlation between MS and CVD is a much-extended field of research on cardiovascular pathology that is gaining more and more attention by scientists (with more than 75000 publications on this topic). In particular, MS and inflammation are linked to myocardial infarction and ischemic heart disease occurrence [59]. Here we will focus our discussion on the role of NLRP3 inflammasome in myocardial ischemia.

In 2001 it has been observed for the first time that the secretion of IL-1 $\beta$ and IL-18 was increased in an I/R model of suprafused human atrial myocardium, thus providing a link between inflammasome activation and CVD development [60]. Since then on, the overexpression of the inflammasome complex has been detected in different models of myocardial I/R during the infarct process. This overexpression was observed in cardiac fibroblasts and infiltrating cells, but also in cardiomyocytes of the border zone of the infarction in post-ischemic myocardium [61,62]. In conditions of MS and diabetes, TXNIP, which is typically upregulated by hyperglycemia, may mediate NLRP3 inflammasome activation in cardiac microvascular endothelial cells as a novel mechanism in myocardial I/R injury [63]. 
Indeed, the block of TXNIP/NLRP3 signaling can inhibit the activation of NLRP3 inflammasomes and may be an interventive strategy to improve myocardial I/R outcome.

It is likely that the formation of the NLRP3 inflammasome in the myocardium during acute heart ischemia may amplify the inflammatory response, thus mediating further injury. It seems that the signaling pathways regulating the inflammasome formation in the cardiac tissue require a priming signal (transcription of inflammasome components) to allow an active NLRP3 to mediate caspase-1 activation and LV dysfunction. Ischemia/reperfusion injury induces both a priming signal and a trigger (NLRP3 activation). Therefore, NLRP3 inflammasome may be active in the ischemic heart to favor caspase-1 activation, inflammation and cell death [64].

It seems that triggers for the NLRP3 inflammasome complex are similar to those responsible for myocardial I/R injury and perhaps to those involved in ischemic preconditioning (PreC). For these reasons, Zuurbier et al. [65] tested the hypothesis that NLRP3 inflammasome plays a role either in PreC or in acute I/R injury of the heart. To this aim they used Langendorff-perfused hearts from wild-type and NLRP3 $3^{(-/)}$mice. Surprisingly, the deletion did not affect I/Rinduced cell death, but exacerbated I/R-induced mechanical dysfunction, and abolished the protective effects of PreC against I/R injury. Since NLRP3 ${ }^{(--)}$hearts had lower levels of IL-6 and lower signal transducer and activator of transcription (STAT)3 expression after PreC, the authors suggested that the observed effects are due to an altered IL6/STAT3 dependent mechanism.

Moreover, Hermansson et al. [66] studied how NLRP3 molecules are influenced in human ischemic cardiac tissue. Intriguingly, NLRP3 levels were markedly low in human ischemic myocardial tissue compared with nonischemic control cardiac tissue. However, gene analysis revealed mutations in NLRP3 in human heart tissues from ischemic subjects, but not in those from non-ischemic controls. The authors concluded that genetic defects in the inflammasome and associated proteins may represent a background for promoting ischemic cardiac disease.

A little at variance with above studies, Sandanger et al. [67] found that hearts isolated from NLRP3 $3^{(-/)}$mice, displayed a marked improvement of mechanical function and reduced injury after an I/R protocol, when compared with the responses of wild-type hearts to I/R challenge. Moreover, NLRP3 inflammasome resulted up-regulated in myocardial fibroblasts of wild-type hearts few days after a permanent coronary occlusion. Thus, the authors suggested that the NLRP3 upregulation may be an important contributor to infarct size development during ischemia.

Of note, both the study by Zuurbier et al. [65] and the study by Sandanger et al. [67] suggest important inflammasome-independent effects of myocardial NLRP3, as these two studies report a clear difference in phenotypes comparing NLRP3 ${ }^{(-)}$and ASC ${ }^{(--)}$hearts. However, and surprisingly enough, the latter group of researchers [68] in a recent study observed a larger infarct size in $\mathrm{NLRP}^{(--)}$and $\mathrm{ASC}^{(--)}$mice (no difference in the two $\mathrm{KO}$ mice), suggesting a cardioprotective role for the NLRP3 inflammasome in myocardial I/R injury, and these authors conclude: "Our data imply that NLRP3 contributes to cardio-protection during I/R and do not support a role for NLRP3 or ASC inhibition in the management of acute myocardial infarction including revascularization therapy". Yet, as noted by Toldo et al. [34], these conclusions set forth by Sandanger et al. [68] are not completely supported by the data of the article, in which, for example, troponin I levels, used as marker of myocardial injury, at $24 \mathrm{~h}$ of reperfusion were similar in the treated and control groups. In this line is also our recent report [33], in which we demonstrated that the heart of mice fed with a high-fat high-fructose diet for 12 weeks had a marked overexpression of both NLRP3 and markers of oxidative metabolism, and a downregulation of both the hypoxia inducible factor (HIF)- $2 \alpha$ and the factors of the RISK pathway. When exposed to cardiac ex vivo $\mathrm{I} / \mathrm{R}$ protocol these hearts displayed a greater infarct size and lactic dehydrogenase release in comparison with control mice. The majority of above reported studies display a role for NLRP3 activation in the exacerbation of myocardial I/R injury. However, the discrepancies in the above reported 
investigations underscore the need for further studies to elucidate the role for NLRP3 inflammasome within the heart. Moreover, pharmacological studies are necessary to determine, among other things, mechanisms of action of the tested drugs and tissue and serum drug levels after local or systemic administration.

\subsection{Liver: NLRP3 inflammasome in steatohepatitis, an overview}

The typical hepatic outcome of MS and over-nutrition is the nonalcoholic fatty liver disease (NAFLD), where the increasing deposition of intracellular lipids may evolve in steatohepatitis (NASH) and fibrosis [69]. Several factors contribute to the progression from NAFLD to NASH, and the currently hypothesized mechanism is based on a "twohit" hypothesis. The first hit includes steatosis and insulin resistance, and these conditions seem to sensitizes the liver for later events leading to the development of steatohepatitis. The second hit is a multifactorial process including oxidative stress, lipid peroxidation, and mitochondrial dysfunction that concur to the chronic inflammatory response [70,71], as occurring in metaflammation. Around 10-20\% patients with NAFLD will eventually develop NASH [71], which has been predicted to be the leading indication for liver transplantation in the USA by the year 2020 [72].

Recent studies in murine models and in human patients with NASH reviewed by Wan and colleagues [73] have evidenced the increased expression of NLRP3 and demonstrated that genetic deletion or pharmacological inhibition of genes related to NLRP3 resulted in alleviation of hepatic steatosis, inflammation, and fibrosis, suggesting that NLRP3 inflammasome may play a critical role in the development of NASH and may act as a molecular therapeutic target. However, the mechanisms linking MS-related hepatosteatosis to NLRP3 activation and further progression to NASH are not fully understood.

In particular, the most likely factor triggering hepatic inflammation in MS is the lipotoxicity induced by the excessive flux of free fatty acids through hepatocytes [74] resulting in accumulation of saturated fatty acids, as palmitate, and sphingolipid metabolism intermediates as ceramide [74,75]. In fact, both palmitate and ceramide have been ascribed for NLRP3 inflammasome activation in in vitro and in vivo studies [35,75]. In a work by Sui et al. [76], a high fat diet was able to induce in mice hepatosteatosis and inflammasome activation, but when the diet was enriched by polyunsaturated fatty acids, the liver showed decreased sensitization to lipopolysaccharide (LPS)-induced NLRP3 inflammasome activation. In particular, authors showed that palmitic acid was able to directly activate NLRP3 inflammasome in cultured primary hepatocytes, while the polyunsaturated docosahexaenoic acid inhibited LPS-induced NLRP3 activation.

In the development of steatohepatitis the resident macrophages are critical in maintaining tissue homeostasis, regulating lipid metabolism, energy homeostasis, and tissue remodeling. An elegant in vitro study on bone marrowderived macrophages revealed that ceramide exposition causes activation of caspase-1. Notably, both caspase-1 activation and IL-1 $\beta$ secretion from macrophages induced by ceramide were blocked in the absence of Nlrp3 [35], suggesting that accumulation of ceramide may be a potent direct inducer of inflammasome activation also in hepatic macrophages.

In MS, the accumulation of palmitate and ceramide is often reported as a typical result of an excessive de novo lipid synthesis due to a deregulated activation of the transcription factor sterol regulatory element-binding protein SREBP1c which regulate the transcription of the enzymes involved in lipogenesis [77,78]. In this contest, there is emerging evidence that AGEs deriving from high fats and sugars intake can interfere not only with SREBP activity, but also with sphingolipids metabolism leading to the specific accumulation of palmitate and ceramide, respectively [79,80]. Moreover, literature data indicate that SREBP itself can induce NLRP3 transcription in atherosclerotic plaques [81-83] and a reciprocal activation between SREBP and NLRP3 is reported in liver with hepatitis C infection [84]. 
Indeed, the inhibition of TNXIP by quercetin and allopurinol leads to the concomitant downregulation of SREBP and NLRP3 activities in the liver of streptozotocin-induced diabetic rats [85]. Taken together, the above-mentioned findings confirm the tight relationship between an unbalanced lipid metabolism with NLRP3 inflammasome activation in the liver.

A further interesting observation concerning the role of diet-derived AGEs in MS-related NLRP3 activation is that the AGEs receptors RAGE and galectin-3 seem to be involved in inflammasome modulation in macrophages. In particular RAGE is suggested to exert an indirect action on NLRP3 as it prepares its activation by promoting the transcription of pro-caspase-1 and pro-IL1 $\beta$ that are secreted in a second time [55]. Otherwise, the upregulated expressions of galectin-3 and NLRP3 were directly correlated in the liver of patients with primary biliary cholangitis and in a mouse model of autoimmune cholangitis, with subsequent activation of caspase- 1 and IL- $1 \beta$ secretion. In the same study, the hepatic activation of NLRP3 was impaired and inflammation significantly reduced when galectin-3 was knocked out in the autoimmune cholangitis model [86]. In regards to MS, a previous interesting work demonstrated that the ablation of galectin-3 in mice put on a $60 \%$ fat diet leads to a worsening of lipids accumulation within hepatocytes compared to the wild type mice fed the same diet, but this enhanced steatosis was paralleled by improvement of the inflammatory profile with downregulated NLRP3 and IL-1 $\beta$ expression, and reduced fibrosis and cell damage [87].

Despite a substantial number of studies indicating the prominent role of macrophages in NLRP3-induced hepatic inflammation, an interesting study evidenced that NLRP3 activation in steatohepatitis can also occur in hepatocytes, in addition to immune cells [88]. Indeed, the selective deficiency of myeloid differentiation primary response 88 (MyD88) gene, one of the main transducers of DAMPs signaling leading to NLRP3 complex assembling, either on bone marrowderived or non-bone marrow-derived cells, downregulates NLRP3 expression, caspase-1 activation, and IL-1 $\beta$ production. However, an attenuation of liver injury was detected only when MyD88 was deleted in macrophages [88].

\subsection{Kidney: NLRP3 inflammasome in diabetic nephropathy, an overview}

The finding that diabetic patients have high levels of IL-18 in plasma and urine which positively correlate with albumin excretion suggested an involvement of inflammasome in diabetic nephropathy [89]. An elegant study on an animal model of diabetic nephropathy showed that increased renal NLRP3 expression and IL-1 $\beta$ and IL-18 levels preceded albuminuria, mesangial cells expansion and glomerulosclerosis onset, that were prevented by NLRP3 deficiency, independently from glucose control, confirming the direct causative role of inflammasome in nephropathy [90]. In the same study, it has been demonstrated through transplantation of NLRP3-deleted bone marrow cells in db/db mice or wild type bone marrow cells in NLRP3-deleted streptozotocin-diabetic mice, that the activation of NLRP3 signaling in renal resident cells has a greater impact than that of infiltrating immune cells in early stages of nephropathy development [90]. In particular, recent literature data have evidenced that NLRP3 is expressed and potentially activated in kidney diseases in different renal resident cells, such as podocytes, glomerular endothelial cells, and tubular interstitial epithelial cells [90,91].

It is widely demonstrated that besides the onset of hepatosteatosis and cardiovascular diseases, the MS confers also increased risk for chronic kidney disease (CKD) [92]. As so far evidenced, dyslipidemia is the MS-related factor mainly responsible for cardiovascular risk and hepatic inflammation, while the role of lipid metabolism in the onset of CKD is still under debate. However, almost all the elements above described for cardiac and hepatic outcomes of lipid metabolism disturbances have been shown to have a role in renal disease development. Indeed, lipotoxicity induced by intracellular lipid accumulation (including saturated fatty acids and ceramide), altered lipid synthesis due to SREBP over-activation, and lipid peroxidation inducing AGEs production, have been recognized as leading causes of 
glomerulosclerosis and tubulo-interstitial damage in animal models of diabetes [93,94]. In this regard, a very recent study has demonstrated the beneficial effect of sphingomyelinase ablation that, by reducing the high-fat diet-induced ceramide production, prevents NLRP3 inflammasome complex formation and subsequent IL-1 $\beta$ production in podocytes, attenuating glomerular damage and proteinuria [95].

Thus, even in kidney the lipotoxicity-driven inflammation and fibrosis are underlying mechanisms of tissue damage [93] and the potential role of NLRP3 inflammasome in diet-induced metaflammation in kidney is currently intensively investigated.

An interesting work comparing the effects of a western-style diet and high-fructose drinking in wild type and NLRP3 knockout mice demonstrated that the activation of NLRP3 signaling is fundamental for the renal macrophagic infiltration and profibrotic signal in diet-induced nephropathy. Moreover, the NLRP3 deletion significantly prevented renal lipids accumulation by downregulating SREBPs activation, confirming a tight relation between lipid synthesis and NLRP3. Furthermore, NLRP3 knockout mice had also strongly reduced hyperuricemia and albumin excretion, especially those fed the western-diet [96].

Hyperuricemia is an important aspect of MS associated to the progression of renal failure [97]. Indeed, uric acid has been demonstrated by in vitro studies to exert a pro-inflammatory action on endothelial and epithelial tubular cells promoting the release of HMGB1 that stimulates the TLR4 and the assembling of NLRP3 inflammasome platform in recruited macrophages $[98,99]$. Indeed, the reduction of circulating uric acid by allopurinol on high fat diet-induced hyperuricemia in a rat model of type 2 diabetes also led to attenuated renal infiltration of macrophages with activated NLRP3 and secreting IL-1 $\beta$, and overall improved nephropathy, even in absence of glycemic control [99].

However, in addition to lipotoxicity, another proposed mechanism for inflammasome activation in renal cells in diabetes and MS, is linked to hyperglycemia that through enhanced glycolytic flux in mitochondria promotes ROS, AGEs, and ROS-mediated TXNIP production, all potential activators of inflammasome platform [37,100,101]. In particular, the AGE-RAGE axis has been found to enhance the podocytes expression of NLRP3 and cleavage of IL-1 $\beta$ [100].

Previous studies have found in the glomeruli of diabetic rats and in a model of inflammatory acute renal injury an up-regulation of the purinergic $2 X 7$ receptor (P2X7R), characterized by a dual response to extracellular ATP [102]. Interestingly, the ablation of P2X7R in high-fat diet fed mice reduced the accumulation of AGEs from both glycation and lipoxidation reactions, and the activation of AGE-RAGE signaling, with parallel downregulation of NLRP3 activation and all the inflammasome components expression. The potential of the targeting of P2X7R-NLRP3 axis in renal disease of type 2 diabetes and metabolic disorders was also confirmed by analysis on human kidney biopsies showing increased expression of P2X7R and NLRP3 in diabetic patients compared to non-diabetic [103].

In addition, high glucose levels in cultured podocytes, as well as in streptozotocin-diabetic mice, induced accumulation of TXNIP, which played a crucial role in NLRP3 inflammasome activation promoting podocyte and glomerular inflammatory injury [101]. Indeed, the inhibition of TXNIP by continuous delivering of TXNIP DNAzyme in (mRen-2)27 transgenic rats rendered diabetic by streptozotocin injection, a model of progressive diabetic nephropathy, improved oxidative stress, reduced NLRP3 expression, and ameliorated fibrosis within the tubulointerstitium [104]. 

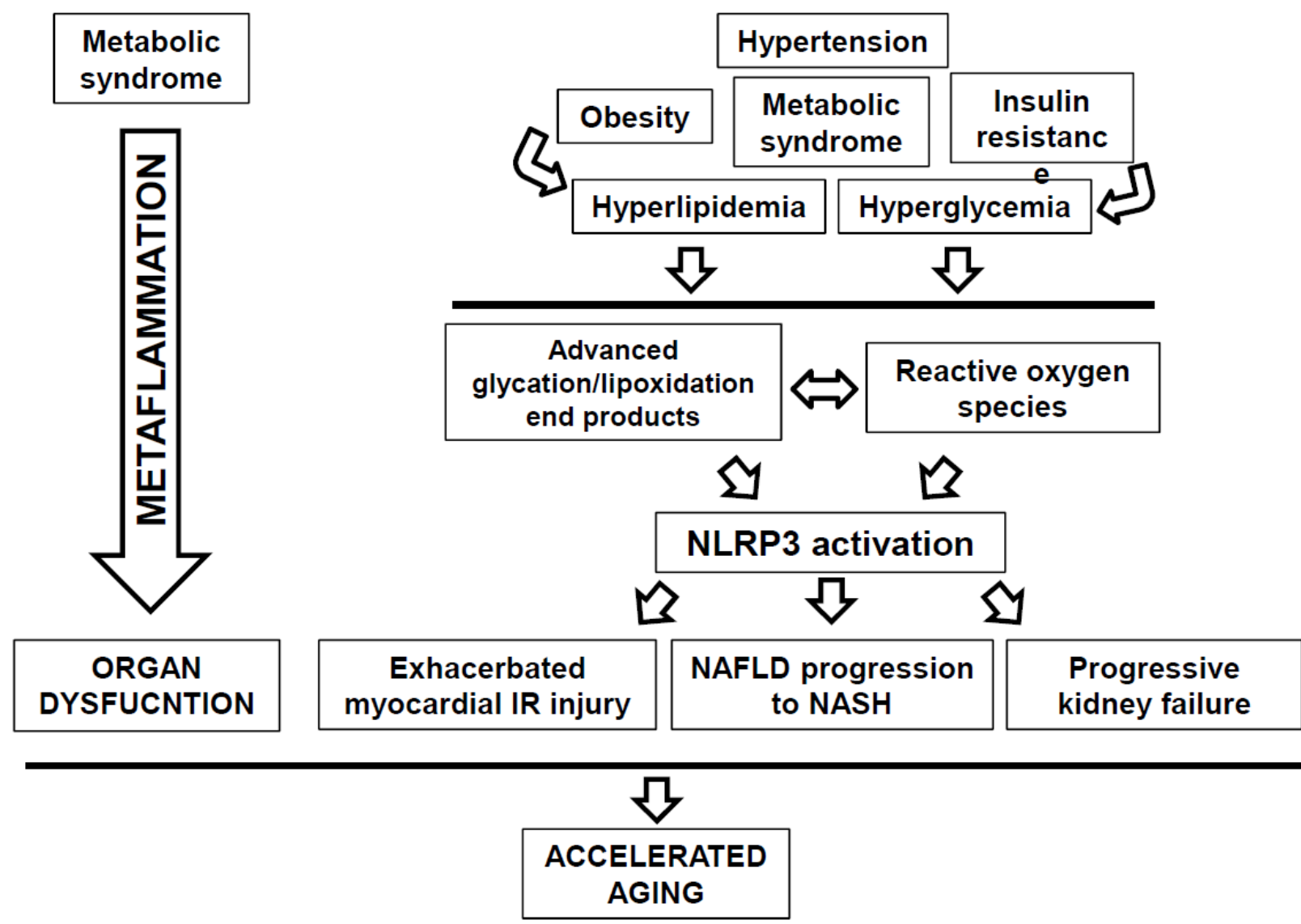

Figure 2. Schematic illustration of metaflammation linking the cluster of factors defining the metabolic syndrome to progressive organ dysfunction. In particular, hyperlipidemia and hyperglycemia are responsible for the accumulation of oxidative stress and toxic compounds as glycation and lipoxidation products that can directly or indirectly activate the NLRP3 inflammasome, the key effector of dysmetabolism-induced tissue damage. Since AGEs, ROS and low chronic inflammation due to reduced innate immune response are common features of the physiological ageing, the metabolic syndrome and the related metaflammation process may represent a kind of accelerated ageing.

\section{THERAPEUTIC INTERVENTIONS TARGETING NLRP3 ACTIVATION IN METAFLAMMATION.}

Several therapeutic approaches have been undertaken in the attempt to both deepen the comprehension of NLRP3 involvement in metaflammation and evaluate whether the targeting of NLRP3 in metabolic diseases might be a promising strategy of intervention.

In regards to the NLRP3 activity in the heart, in a recent study Marchetti et al [105] demonstrated an increased caspase-1 activity and cell death in HL-1 cardiomyocytes treated with lipopolysaccharide (NLRP3 priming) and ATP (NLRP3 trigger). The increased NLRP3 activity was abolished by 16673-34-0, a new synthesized inhibitor of the NLRP3 inflammasome. Moreover, pre-treatment with this inhibitor in a model of acute I/R, a model similar to that of Sandanger et al. [68], blunted the activity of caspase-1 and reduced cardiac infarct size. This is in line with a previous study of the same group [62] in which the authors showed that the inhibition of the inflammasome by small-interfering RNA prevents NLRP3 activation and cardiac cell death, resulting in an improvement of cardiac remodeling. In such a study, they suggested that inflammasome activation within cardiomyocytes promotes caspase-1-dependent cardiomyocyte death, termed 'pyroptosis'.

We have recently confirmed $\mathrm{w}$ that the inhibition of NLRP3 activation by acute administration of a novel small electrophilic molecule limits the extension of infarct size [106]. In addition, Toldo et al [107] found that only if given 
within $1 \mathrm{~h}$ of reperfusion the NLRP3 inflammasome inhibitor, 16673-34-0 [108], limits the secondary inflammatory and infarct size injury that usually follow myocardial I/R. Yet, Jong et al. [109] showed no differences in cardiac damage between NLRP3(-/-) and wild-type mice in a model of closed-chest myocardial I/R injury. Finally, it has been reported that selective inhibition of the NLRP3-inflammasome reduces infarct size and preserves cardiac function also in a large animal MI model. Indeed, a 7 days treatment with the NLRP3-inflammasome inhibitor MCC950 significantly reduced infarct size and preserved left ventricular ejection fraction in landrace pigs subjected to 75 min transluminal balloon occlusion. Moreover, MCC950 treatment reduced myocardial neutrophil influx and IL-1 $\beta$ levels. These results further confirm the therapeutic potential of NLRP3-inflammasome inhibition in acute MI patients [110].

As said above, a number of studies have reported that regulating NLRP3 inflammasome activation with small molecule inhibitors is a potential therapeutic strategy to treat or prevent the pathological outcomes of NAFLD [30,31,111]. As above mentioned, the activation of NLRP3 in the liver is strictly correlated to the intrahepatic accumulation of lipids and thus the inhibition of TNXIP by quercetin and allopurinol leads to the concomitant downregulation of SREBP and NLRP3 activities in the liver of streptozotocin-induced diabetic rats [85]. In a very recent work, the potential prevention of NAFLD in the liver of high fat fed mice by sulforaphane, an organosulfur compound that exhibits potent anti-inflammatory activity and attenuates diet-induced adiposity and hepatosteatosis, has been investigated [112]. The results demonstrate that sulforaphane suppresses NLRP3 inflammasome activation in hepatocytes preventing hepatic steatosis. Moreover, since the mitochondrial dysfunction is the preceding event occurring with saturated fat-induced activation of the NLRP3 inflammasome [37], the same study showed that sulforaphane stimulates the autophagic process that recover the mitochondrial dysfunction induced by palmitic acid in cultured hepatocytes, thereby downregulating fat-induced activation of the NLRP3 inflammasome through AMPKautophagy signaling [112].

An interesting natural compound extensively investigated for its beneficial effects mediated by antioxidant and anti-inflammatory properties is resveratrol. Resveratrol is a polyphenol found in grape, mulberry and red wine, that has been shown to exert cardio-protective and anti-cancer effects [113,114]. Results from in vitro and animal experimental models, confirmed by a human clinical trial in diabetic subjects, demonstrated the ability of resveratrol to improve insulin resistance and diabetes [115]. In a recent work, four weeks of resveratrol administration improved glycemic control and hepatic steatosis in a diet-induced obesity rodent model (10 weeks of $45 \%$ high-fat diet) by inhibiting the activation of NLRP3 inflammasome and hepatic metaflammation. The activation of AMPK through increase in Sirt-1 and Sirt-6, and the increased production of the anti-inflammatory cytokine adiponectin, are suggested as the underlying mechanisms for the beneficial effects of resveratrol on NLRP3 inflammatory signaling [116].

In addition to resveratrol, other polyphenols can provide modulation of innate immune response in obesityassociated NAFLD, as reported by Zou and colleagues [117] who demonstrated the beneficial effect of punicalagin, one of the major ellagitannins found in promegranate, on inflammatory markers in the liver of high fat diet-fed rats. In particular, punicalagin has been reported to selectively inhibit ATP-induced IL-1 $\beta$ release, but not its processing, in NLRP3activated macrophages [118]. Moreover, Tang et al. [119] demonstrated that 4-weeks administration of ellagic acid ameliorated monocrotaline-induced pulmonary artery hypertension in rats by inhibiting NLRP3 inflammasome signal pathway in lungs.

Another approach aimed to prevent NLRP3 activation in diet-induced hepatosteatosis is the administration of a peroxisome proliferator-activated receptor (PPAR)- $\delta$ activator, as shown in a study from Lee and colleagues [120]. In this study, the PPAR- $\delta$ agonist GW501516 was able to reduce hepatic steatosis, inflammation, and oxidative stress in mice fed a high fat diet, although without affecting insulin resistance and hyperlipidemia. In particular, GW501516 
treatment increased both in vivo and in vitro the phosphorylation of AMPK, which is known to have an antiinflammatory effect and suppress ROS production. Since in immune cells the AMPK-ROS signaling pathway is associated with the activation of the NLRP3 inflammasome, the activation of AMPK may be the potential mechanism underlying the inhibitory effect of PPAR- $\delta$ agonist on NLRP3 [120].

The fact that PPAR- $\delta$ plays important roles in the regulation of metabolism and attenuation of NLRP3 inflammatory pathway, has been firstly evidenced in a mouse model of high-fructose corn syrup-induced dysmetabolism, where the administration of the PPAR- $\delta$ agonist GW0742 ameliorated systemic glucose and lipid metabolism, as well as hepatic insulin resistance, by preventing NLRP3 activation in the kidney [30].

Finally, very recent findings have highlighted new therapeutic potential of the sodium glucose cotransporter (SGLT)-2 inhibitor empagliflozin, currently employed as oral hypoglycemic drug for the treatment of type 2 diabetes. Indeed, empagliflozin attenuated the metabolic abnormalities induced by chronic exposure to a high-fat high-sucrose diet decreasing the hepatic and renal tissue injury associated with obesity and insulin resistance, but interestingly, empagliflozin reduced in a dose-dependent manner the diet-induced activation of NLRP3 inflammasome pathway in both liver and kidney [121].

In addition, as above seen for the liver, also in kidney the same mechanism related to TXNIP inhibition is involved in the positive effects exerted by several natural compounds provided with antioxidant properties on renal function. In fact, compounds such as piperine, an active alkaloidal phenolic component of black pepper, cepharanthine, a biscoclaurine alkaloid isolated from Stephania cepharantha Hayata, pterostilbene, a natural dimethylether derivative of resveratrol, or the Simiao pill, a famous traditional Chinese medicine formula, preserved renal function in animal models of streptozotocin- and diet-induced diabetes and MS by reducing NLRP3 activation through prevention of ROSinduced TXNIP production [122-124].

Among the numerous promising strategies for the preservation of kidney function in MS and diabetes through NLRP3 inhibition, we have previously cited the positive results of PPAR $\delta$ agonists and SGLT-2 inhibitor in both hepatic and renal tissue [30,121]. It can be mentioned also a study on a dipeptidyl peptidase (DPP) 4 inhibitor, saxagliptin which was found to attenuated diabetes-induced activation of the inflammasome and progression of diabetic nephropathy in two models of type 1 (Akita mice) and type 2 (BTBR mice) diabetes. In particular, the effects of saxagliptin in the Akita mice were independent of glucose lowering [125].

A novel and promising treatment option for MS-related disease is the use of selective inhibitors of the NLRP3 inflammasome. To date, no specific and selective compounds are available for the inhibition of inflammasome activation in MS. However, a very recent study highlighted that the pharmacological inhibition of the inflammasome by the use of the selective NLRP3 inhibitor BAY 11-7082, given to mice only for the last 7 wks of the 12-wks of high-fat high-sucrose diet, reversed the deleterious effects of diet exposure preventing the development of insulin resistance and attenuating in both liver and kidney markers of fibrosis and inflammation, thus preserving organ dysfunction [30].

\section{CONCLUSIONS}

Here we provide evidences that NLPR3 inflammasome activation, in which ROS formation may play a pivotal role, is involved in several pathological conditions related with MS. Therefore, it is likely that oxidative stress and NLRP3 inflammasome activation may be common pathogenic denominators linking metaflammation to MS. NLRP3 inflammasome activation is also involved in the exacerbation of myocardial $\mathrm{I} / \mathrm{R}$ injury and prophylactic or postischemic treatments with selective NLRP3 inhibitor limit I/R damage. These findings convincingly support its role as cross-talk mechanism in cardio-metabolic diseases. The tight relationship between lipid metabolism unbalance and 
NLRP3 inflammasome activation in the liver seems to be the triggering factor for the progression of NAFLD to NASH in MS. Indeed, many strategies aimed at the modulation of lipid synthesis also limit the NLRP3 activation, and viceversa, when acting a direct inhibition of NLRP3 the lipid metabolism is improved as consequence.

Besides the dysregulated lipid metabolism, the hyperglycemia, often present in MS, has an important role in the inflammatory response and specifically in NLRP3 activation leading to renal dysfunction. In particular, hyperglycemia in the kidney is strictly related to oxidative stress and AGEs accumulation, which, as extensively demonstrated, play a crucial role in NLRP3 activation and chronic inflammation. Any strategy targeting the reduction of oxidative stress or the inhibition of AGEs accumulation or AGE-RAGE signaling exert a positive effect on kidney function through the modulation of NLRP3 activation.

In summary, in the present review recently discovered characteristics of NLRP3 activity and regulation are highlighted as far as they might lead to selective intervention by specific drugs. Indeed, pharmacological modulation of NLRP3 inflammasome has the potential to exert beneficial effects in the control of metabolic dysregulation and its cardiovascular, hepatic and renal complications. Drugs targeting NLRP3 may thus represent unique tools in therapeutic armamentarium against MS-related diseases. They could be protagonists in the strategies aimed to address the burden of cardiovascular, kidney and liver-related morbidity and mortality, due to the co-presence of MS, obesity and insulin resistance. Clearly, there is a stringent need to dedicate considerable effort in understanding such pathological conditions and therapeutic potential if we are to "bridge the gap". A hope of progress comes from the development of new promising technologies, including the so-called omic sciences, the investigations of ROS-dependent pathways, and the studies on the role of stem and progenitor cells. In this perspective, animal studies on models of risk factors for CVD and comorbidities, such as those correlated to MS, are essential for adequate successive clinical translation. Basic and translational studies may help to clarify how effective is a therapeutic strategy which include NLRP3 inhibitors in order to potentially prevent and cure metaflammation-mediated diseases.

\section{LIST OF ABBREVIATIONS}

AGEs, advanced glycation endproducts; AMPK, AMP-activated protein kinase; ASC, apoptosis-associated speck-like protein including a caspase recruitment domain; CKD, chronic kidney disease

CLRs, C-type lectin receptors; CVD, cardiovascular diseases; DAMPs, damage-associated molecular patterns; DPP, dipeptidyl peptidase; HIF-2 $\alpha$, hypoxia inducible factor-2 $\alpha$; I/R, ischemia/reperfusion; IL, interleukin; LPS, lipopolysaccharide; MS, metabolic syndrome; MyD88, myeloid differentiation primary response 88; NAFLD, nonalcoholic fatty liver disease; NASH, steatohepatitis; NF- $\kappa \mathrm{B}$, nuclear factor- $\kappa \mathrm{B}$; NLRP3, NOD-like receptor pyiridin domain containing 3; P2X7, purinergic receptor 7; PAMPs, pathogen-associated molecular patterns; PPAR, peroxisome proliferator-activated receptor; PreC, preconditioning; PRRs, pattern recognition receptors; RAGE, receptor for AGEs; RISK, Reperfusion Injury Salvage Kinases; RLRs, Retinoic acid-inducible gene (RIG)-I-like receptors; ROS, reactive oxygen species; SAFE, Survivor Activating Factor Enhancement; SGLT, sodium glucose cotransporter; STAT3, signal transducer and activator of transcription 3; TAK1, transforming growth factor $\beta$-activated kinase 1 ; TLRs, Toll-like receptors; TNF- $\alpha$, tumor necrosis factor- $\alpha$; TNXIP, thioredoxin-interacting protein; TRP, transient receptor potential.

\section{CONFLICT OF INTEREST}

Authors have no conflict of interests to declare 


\section{References}

[1] Bonomini, F.; Rodella, L.F.; Rezzani, R. Metabolic Syndrome, Aging and Involvement of Oxidative Stress. Aging and Disease, 2015, 6(2), $109-120$.

[2] Jura, M.; Kozak, L.P. Obesity and related consequences to ageing. Age, 2016, 38, 23-41.

[3] Fakhouri, T.H.; Ogden, C.L.; Carroll, M.D.; Kit, B.K.; Flegal, K.M. Prevalence of obesityamong older adults in the United States, $2007-2010$. NCHS Data Brief, 2012, 106, 1-8.

[4] Eckel, R.H.; Grundy, S.M.; Zimmet, P.Z. The metabolic syndrome. Lancet, 2005, 365, 1415-28.

[5] Portincasa, P.; Grattagliano, I.; Palmieri, V.O.; Palasciano, G. Nonalcoholic steatohepatitis: recent advances from experimental models to clinical management. Clin. Biochem., 2005, 38, 203-217.

[6] Despres, J.P.; Lemieux, I. Abdominal obesity and metabolic syndrome. Nature, 2006, 444, 881-7.

[7] Redon, J.; Lurbe, E. The kidney in obesity. Curr. Hypertens. Rep., 2015, 17(6), 555.

[8] Fernández-García, J.C.; Cardona, F.; Tinahones, F.J. Inflammation, oxidative stress and metabolic syndrome: dietary modulation. Curr. Vasc. Pharmacol., 2013, 11(6), 906-19.

[9] Dyer, D.G.; Dunn, J.A.; Thorpe, S.R.; Bailie, K.E.; Lyons, T.J.; McCance, D.R.; Baynes, J.W. Accumulation of Maillard reaction products in skin collagen in diabetes and aging. J. Clin. Invest., 1993, 91, 2463-9.

[10] Hu, S.; He, W.; Liu, Z.; Xu, H.; Ma, G. The accumulation of the glycoxidation product N(epsilon)-carboxymethyllysine in cardiac tissues with age, diabetes mellitus and coronary heart disease. Tohoku J. Exp. Med., 2013, 230(1), 25-32.

[11] Shaw, A.C.; Goldstein, D.R.; Montgomery, R.R. Age-dependent dysregulation of innate immunity. Nat. Rev. Immunol., 2013, 13(12), 875887.

[12] Reynaert, N.L.; Gopal, P.; Rutten, E.P.; Wouters, E.F.; Schalkwijk, C.G. Advanced glycation end products and their receptor in age-related, non-communicable chronic inflammatory diseases; Overview of clinical evidence and potential contributions to disease. Int. J. Biochem. Cell. Biol., 2016, S1357-2725(16), 30156-X.

[13] Ahima, R.S. Connecting obesity, aging and diabetes. Nat. Med., 2009, 15(9), 996-997.

[14] Ribeiro Filho, F.F.; Mariosa, L.S.; Ferreira, S.R.; Zanella, M.T. Visceral fat and metabolic syndrome: more than a simple association. Arq. Bras. Endocrinol. Metabol., 2006, 50(2), 230-8.

[15] Hotamisligil, G.S. Inflammation and metabolic disorders. Nature, 2006, 444(7121), 860-7.

[16] Charrière, G.; Cousin, B.; Arnaud, E.; André, M.; Bacou, F.; Penicaud, L.; Casteilla, L. Preadipocyte conversion to macrophage. Evidence of plasticity. J. Biol. Chem., 2003, 278(11), 9850-5.

[17] Wellen, K.E.; Hotamisligil, G.S. Inflammation, stress, and diabetes. J. Clin. Invest., 2005, 115(5), 1111-9.

[18] Ghosh, A.R., Bhattacharya, R., Bhattacharya, S., Nargis, T., Rahaman, O., Duttagupta, P., Raychaudhuri, D., Liu, C.S., Roy, S., Ghosh, P., Khanna, S., Chaudhuri, T., Tantia, O., Haak, S., Bandyopadhyay, S., Mukhopadhyay, S., Chakrabarti, P., Ganguly, D. Adipose Recruitment and Activation of Plasmacytoid Dendritic Cells Fuel Metaflammation. Diabetes, 2016, 65(11), 3440-52.

[19] Lee, B.C.; Lee, J. Cellular and molecular players in adipose tissue inflammation in the development of obesity-induced insulin resistance. Biochim. Biophys. Acta., 2014, 1842(3), 446-62.

[20] Van Gaal, L.F.; Mertens, I.L.; De Block, C.E. Mechanisms linking obesity with cardiovascular disease. Nature, 2006, 444(7121), 875-80.

[21] Rydén, L.; Grant, P.J.; Anker, S.D.; Berne, C.; Cosentino, F.; Danchin, N.; Deaton, C.; Escaned, J.; Hammes, H.P.; Huikuri, H.; Marre, M.; Marx, N.; Mellbin, L.; Ostergren, J.; Patrono, C.; Seferovic, P.; Uva, M.S.; Taskinen, M.R.; Tendera, M.; Tuomilehto, J.; Valensi, P.; Zamorano, J.L. Task Force on diabetes, pre-diabetes, and cardiovascular diseases of the European Society of Cardiology (ESC); European Association for the Study of Diabetes (EASD). ESC guidelines on diabetes, pre-diabetes, and cardiovascular diseases developed in collaboration with the EASD. Diab. Vasc. Dis. Res., 2014, 11(3), 133-73.

[22] Martinon, F.; Burns, K.; Tschopp, J. The inflammasome: a molecular platform triggering activation of inflammatory caspases and processing of pro-IL-1ß. Mol. Cell., 2002, 10, 417-426.

[23] Takeuchi, O.; Akira, S. Pattern recognition receptors and inflammation. Cell, 2010, 140, 805-820.

[24] Takahashi, M. Role of the inflammasome in myocardial infarction. Trends Cardiovasc. Med., 2011, $21,37-41$.

[25] Schroder, K.; Zhou, R.; Tschopp, J. The NLRP3 inflammasome: a sensor for metabolic danger? Science, 2010, 327, 296-300.

[26] Chen, G.Y.; Nuñez, G. Sterile inflammation: sensing and reacting to damage. Nat. Rev. Immunol., 2010, $10(12), 826-837$.

[27] Campbell, M.; Doyle, S.L. An eye on the future of inflammasomes and drug development in AMD. J. Mol. Med., 2013, 91(9), 1059-70.

[28] Drouin-Ouellet, J.; Cicchetti, F. Inflammation and neurodegeneration: the story 'retolled'. Trends Pharmacol. Sci., 2012, 33(10), 542-51.

[29] Salminen, A.; Ojala, J.; Kaarniranta, K.; Kauppinen, A. Mitochondrial dysfunction and oxidative stress activate inflammasomes: impact on the aging process and age-related diseases. Cell. Mol. Life. Sci., 2012, 69(18), 2999-3013. 
[30] Collino, M.; Benetti, E.; Rogazzo, M.; Mastrocola, R.; Yaqoob, M.M.; Aragno, M.; Thiemermann, C.; Fantozzi, R. Reversal of the deleterious effects of chronic dietary HFCS-55 intake by PPAR- $\delta$ agonism correlates with impaired NLRP3 inflammasome activation. Biochem. Pharmacol., 2013, 85(2), 257-64.

[31] Chiazza, F.; Couturier-Maillard, A.; Benetti, E.; Mastrocola, R.; Nigro, D.; Cutrin, J.C.; Serpe, L.; Aragno, M.; Fantozzi, R.; Ryffel, B.; Thiemermann, C.; Collino, M. Targeting the NLRP3 inflammasome to reduce diet-induced metabolic abnormalities in mice. Mol. Med., 2015.

[32] Patel, M.N.; Bernard, W.G.; Milev, N.B.; Cawthorn, W.P.; Figg, N.; Hart, D.; Prieur, X.; Virtue, S.; Hegyi, K.; Bonnafous, S.; Bailly-Maitre, B.; Chu, Y.; Griffin, J.L.; Mallat, Z.; Considine, R.V.; Tran, A.; Gual, P.; Takeuchi, O.; Akira, S.; Vidal-Puig, A.; Bennett, M.R.; Sethi, J.K. Hematopoietic IKBKE limits the chronicity of inflammasome priming and metaflammation. Proc. Natl. Acad. Sci. USA, 2015, 112(2), 506-11.

[33] Mastrocola, R.; Collino, M.; Penna, C.; Nigro, D.; Chiazza, F.; Fracasso V.; Tullio, F.; Alloatti G.; Pagliaro, P.; Aragno, M. Maladaptive Modulations of NLRP3 Inflammasome and Cardioprotective Pathways Are Involved in Diet-Induced Exacerbation of Myocardial Ischemia/Reperfusion Injury in Mice. Oxid. Med. Cell. Longev., 2016, 2016, 3480637.

[34] Toldo, S.; Marchetti, C.; Abbate, A. Re. NLRP3 inflammasome activation during myocardial ischemia reperfusion is cardioprotective. Biochem. Biophys. Res. Commun., 2016, 470(4), 811-2.

[35] Vandanmagsar, B.; Youm, Y.H.; Ravussin, A.; Galgani, J.E.; Stadler, K.; Mynatt, R.L.; Ravussin, E.; Stephens, J.M.; Dixit, V.D. The NLRP3 inflammasome instigates obesity-induced inflammation and insulin resistance. Nat. Med., 2011, 17(2), 179-88.

[36] Ozaki, E.; Campbell, M.; Doyle, S.L. Targeting the NLRP3 inflammasome in chronic inflammatory diseases: current perspectives. J. Inflamm. Res., 2015, 8, 15-27.

[37] Zhou, R.; Yazdi, A.S.; Menu, P.; Tschopp, J. A role for mitochondria in NLRP3 inflammasome activation. Nature, 2011, 469(7329), $221-225$.

[38] Bauernfeind, F.; Bartok, E.; Rieger, A.; Franchi, L.; Nunez, G.; Hornung, V. Cutting edge: reactive oxygen species inhibitors block priming, but not activation, of the NLRP3 inflammasome. J. Immunol., 2011, 187(2), 613-617.

[39] Hornung, V.; Bauernfeind, F.; Halle, A.; Samstad, E.O.; Kono, H.; Rock, K.L.; Fitzgerald, K.A.; Latz, E. Silica crystals and aluminum salts activate the NALP3 inflammasome through phagosomal destabilization. Nat. Immunol., 2008, 9(8), 847-856.

[40] Pelegrin, P.; Surprenant, A. Pannexin-1 mediates large pore formation and interleukin-1beta release by the ATP-gated P2X7 receptor. EMBO $J .$, 2006, 25(21), 5071-5082.

[41] Petrilli, V.; Papin, S.; Dostert, C.; Mayor, A.; Martinon, F.; Tschopp, J. Activation of the NALP3 inflammasome is triggered by low intracellular potassium concentration. Cell. Death. Differ., 2007, 14(9), 1583-1589.

[42] Compan, V.; Baroja-Mazo, A.; Lopez-Castejon, G.; Gomez, A.I.; Martínez, C.M.; Angosto, D.; Montero, M.T.; Herranz, A.S.; Bazán, E.; Reimers, D.; Mulero, V.; Pelegrín, P. Cell volume regulation modulates NLRP3 inflammasome activation. Immunity, 2012, 37(3), 487-500.

[43] Gong, Y.N.; Wang, X.; Wang, J.; Yang, Y.; Li, S.; Yang, J.; Liu, L.; Lei, X.; Shao, F. Chemical probing reveals insights into the signaling mechanism of inflammasome activation. Cell. Res., 2010, 20, 1289-1305.

[44] Sutterwala, F.S.; Haasken, S.; Cassel, S.L. Mechanism of NLRP3 inflammasome activation. Ann. NY Acad. Sci, 2014, 1319, $82-95$.

[45] Mastrocola, R.; Penna, C.; Tullio, F.; Femminò, S.; Nigro, D.; Chiazza, F.; Serpe, L.; Alloatti, G.; Cocco, M.; Bertinaria, M.; Pagliaro, P.; Aragno, M.; Collino, M. Pharmacological inhibition of NLRP3 inflammasome attenuates myocardial ischemia/reperfusion injury by activation of RISK and mitochondrial pathways. Oxid. Med. Cell. Longev., 2016, In press.

[46] Barja, G. The mitochondrial free radical theory of aging. Prog. Mol. Biol. Transl. Sci., 2014, 127, 1-27.

[47] Feldman, N.; Rotter-Maskowitz, A.; Okun, E. DAMPs as mediators of sterile inflammation in aging-related pathologies. Ageing research reviews, 2015, 24(Pt A), 29-39.

[48] Mastrocola, R.; Collino, M.; Rogazzo, M.; Medana, C.; Nigro, D.; Boccuzzi, G.; Aragno, M. Advanced glycation end products promote hepatosteatosis by interfering with SCAP-SREBP pathway in fructose-drinking mice. Am. J. Physiol. Gastrointest. Liver Physiol., 2013, 305, G398-407.

[49] Mastrocola, R.; Collino, M.; Nigro, D.; Chiazza, F.; D'Antona, G.; Aragno, M.; Minetto, M.A. Accumulation of Advanced Glycation EndProducts and Activation of the SCAP/SREBP Lipogenetic Pathway Occur in Diet-Induced Obese Mouse Skeletal Muscle. PLoS One, 2015, 10, $\mathrm{e} 0119587$.

[50] Gaens, K.H.; Goossens, G.H.; Niessen, P.M.; van Greevenbroek, M.M.; van der Kallen, C.J.; Niessen, H.W.; Rensen, S.S.; Buurman, W.A.; Greve, J.W.; Blaak, E.E.; van Zandvoort, M.A.; Bierhaus, A.; Stehouwer, C.D.; Schalkwijk, C.G. Ne-(carboxymethyl)lysine-receptor for advanced glycation end product axis is a key modulator of obesity-induced dysregulation of adipokine expression and insulin resistance. Arterioscler. Thromb. Vasc. Biol., 2014, 34(6), 1199-208.

[51] Maessen, D.E.; Brouwers, O.; Gaens, K.H.; Wouters, K.; Cleutjens, J.P.; Janssen, B.J.; Miyata, T.; Stehouwer, C.D.; Schalkwijk, C.G. Delayed Intervention With Pyridoxamine Improves Metabolic Function and Prevents Adipose Tissue Inflammation and Insulin Resistance in High-Fat Diet-Induced Obese Mice. Diabetes, 2016, 65(4), 956-66. 
[52] Mastrocola, R.; Nigro, D.; Chiazza, F.; Medana, C.; Dal Bello, F.; Boccuzzi, G.; Collino, M.; Aragno, M. Fructose-derived advanced glycation end-products drive lipogenesis and skeletal muscle reprogramming via SREBP-1c dysregulation in mice. Free Radic. Biol. Med., 2016, 91, 224-35.

[53] Bakala, H.; Ladouce, R.; Baraibar, M.A.; Friguet, B. Differential expression and glycative damage affect specific mitochondrial proteins with aging in rat liver. Biochim. Biophys. Acta., 2013, 1832, 2057-2067.

[54] Cai, W.; Uribarri, J.; Zhu, L.; Chen, X.; Swamy, S.; Zhao, Z.; Grosjean, F.; Simonaro, C.; Kuchel, G.A.; Schnaider-Beeri, M.; Woodward, M.; Striker, G.E.; Vlassara, H. Oral glycotoxins are a modifiable cause of dementia and the metabolic syndrome in mice and humans. Proc. Natl. Acad. Sci. USA, 2014, 111, 4940-4945.

[55] He, Q.; You, H.; Li, X.M.; Liu, T.H.; Wang, P.; Wang, B.E. HMGB1 promotes the synthesis of pro-IL-1 $\beta$ and pro-IL-18 by activation of p38 MAPK and NF-KB through receptors for advanced glycation end-products in macrophages. Asian Pac. J. Cancer Prev., 2012, 13(4), 1365-70.

[56] Kang, R.; Chen, R.; Xie, M.; Cao, L.; Lotze, M.T.; Tang, D.; Zeh, H.J 3rd. The Receptor for Advanced Glycation End Products Activates the AIM2 Inflammasome in Acute Pancreatitis. J. Immunol., 2016, 196(10), 4331-7.

[57] Pejnovic, N.N.; Pantic, J.M.; Jovanovic, I.P.; Radosavljevic, G.D.; Milovanovic, M.Z.; Nikolic, I.G.; Zdravkovic, N.S.; Djukic, A.L.; Arsenijevic, N.N.; Lukic, M.L. Galectin-3 deficiency accelerates high-fat diet-induced obesity and amplifies inflammation in adipose tissue and pancreatic islets. Diabetes, 2013, 62(6), 1932-44.

[58] Steinberg, G.R.; Schertzer, J.D. AMPK promotes macrophage fatty acid oxidative metabolism to mitigate inflammation: implications for diabetes and cardiovascular disease. Immunology and cell biology, 2014, 92, 340-345.

[59] Thomsen, M.; Nordestgaard, B.G. Myocardial infarction and ischemic heart disease in ovrweight and obesity with and without metabolic syndrome. JAMA Intern. Med, 2014, 174(1), 15-22.

[60] Pomerantz, B.J.; Reznikov, L.L.; Harken, A.H.; Dinarello, C.A. Inhibition of caspase 1 reduces human myocardial ischemic dysfunction via inhibition of IL-18 and IL-13. Proc. Natl. Acad. Sci. USA, 2001, 98(5), 2871-2876.

[61] Kawaguchi, M.; Takahashi, M.; Hata, T.; Kashima, Y.; Usui, F.; Morimoto, H.; Izawa, A.; Takahashi, Y.; Masumoto, J.; Koyama, J.; Hongo, M.; Noda, T.; Nakayama, J.; Sagara, J.; Taniguchi, S.; Ikeda, U. Inflammasome activation of cardiac fibroblasts is essential formyocardial ischemia/reperfusion injury. Circulation, 2011, 123(6), 594- 604.

[62] Mezzaroma, E.; Toldo, S.; Farkas, D.; Seropian, I.M.; Van Tassell, B.W.; Salloum, F.N.; et al. The inflammasome promotes adverse cardiac remodeling following acute myocardial infarction in the mouse. Proc Natl Acad Sci USA, 2011, 108, 19725-19730.

[63] Liu, Y.; Lian, K.; Zhang, L.; Wang, R.; Yi, F.; Gao, C.; Xin, C.; Zhu, D.; Li, Y.; Yan, W.; Xiong, L.; Gao, E.; Wang, H.; Tao, L. TXNIP mediates NLRP3 inflammasome activation in cardiac microvascular endothelial cells as a novel mechanism in myocardial ischemia/reperfusion injury. Basic Res. Cardiol, 2014, 109(5), 415.

[64] Toldo, S.; Mezzaroma, E.; McGeough, M.D.; Peña, C.A.; Marchetti, C.; Sonnino, C.; Van Tassell, B.W.; Salloum, F.N.; Voelkel, N.F.; Hoffman, H.M.; Abbate, A. Independent roles of the priming and the triggering of the NLRP3 inflammasome in the heart. Cardiovasc. Res., 2015, 105(2), 203-12.

[65] Zuurbier, C.J.; Jong, W.M.; Eerbeek, O.; Koeman, A.; Pulskens, W.P.; Butter, L.M.; Leemans, J.C.; Hollmann, M.W. Deletion of the innate immune NLRP3 receptor abolishes cardiac ischemic preconditioning and is associated with decreased Il-6/STAT3 signaling. PLoS One, 2012, 7(7), e40643.

[66] Hermansson, C.; Lundqvist, A.; Wasslavik, C.; Palmqvist, L.; Jeppsson, A.; Hultén, L.M. Reduced expression of NLRP3 and MEFV in human ischemic heart tissue. Biochem. Biophys. Res. Commun., 2013, 430(1), 425-8.

[67] Sandanger, Ø.; Ranheim, T.; Vinge, L.E.; Bliksøen, M.; Alfsnes, K.; Finsen, A.V.; Dahl, C.P.; Askevold, E.T.; Florholmen, G.; Christensen, G.; Fitzgerald, K.A.; Lien, E.; Valen, G.; Espevik, T.; Aukrust, P.; Yndestad, A. The NLRP3 inflammasome is up-regulated in cardiac fibroblasts and mediates myocardial ischaemia-reperfusion injury. Cardiovasc, Res., 2013, 99(1), 164-74.

[68] Sandanger, Ø.; Gao, E.; Ranheim, T.; Bliksøen, M.; Kaasbøll, O.J.; Alfsnes, K.; Nymo, S.H.; Rashidi, A.; Ohm, I.K.; Attramadal, H.; Aukrust, P.; Vinge, L.E.; Yndestad, A. NLRP3 inflammasome activation during myocardial ischemia reperfusion is cardioprotective. Biochem. Biophys. Res. Commun., 2016, 469(4), 1012-20.

[69] Marchesini, G.; Bugianesi, E.; Forlani, G.; Cerrelli, F.; Lenzi, M.; Manini, R.; Natale, S.; Vanni, E.; Villanova, N.; Melchionda, N.; Rizzetto, M. Nonalcoholic fatty liver, steatohepatitis, and the metabolic syndrome. Hepatology, 2003, 37(4), 917-23.

[70] Sanyal, A.J.; Campbell-Sargent, C.; Mirshahi, F.; Rizzo, W.B.; Contos, M.J.; Sterling, R.K.; Luketic, V.A.; Shiffman, M.L.; Clore, J.N. Nonalcoholic steatohepatitis: association of insulin resistance and mitochondrial abnormalities. Gastroenterology, 2001, 120(5), 1183-1192.

[71] Tilg, H.; Moschen, A.R. Evolution of inflammation in nonalcoholic fatty liver disease: the multiple parallel hits hypothesis. Hepatology, 2010, 52(5), 1836-1846.

[72] Mahady, S.E.; George, J. Management of nonalcoholic steatohepatitis: an evidence-based approach. Clinical Liver Disease, 2012, 16(3), 631645. 
[73] Wan, X.; Xu, C.; Yu, C.; Li, Y. Role of NLRP3 Inflammasome in the Progression of NAFLD to NASH. Can. J. Gastroenterol. Hepatol., 2016, $2016,6489012$.

[74] Csak, T.; Ganz, M.; Pespisa, J.; Kodys, K.; Dolganiuc, A.; Szabo, G. Fatty acid and endotoxin activate inflammasomes in mouse hepatocytes that release danger signals to stimulate immune cells. Hepatology, 2011, 54(1), 133-44.

[75] Summers, S.A. Ceramides in insulin resistance and lipotoxicity. Prog. Lipid Res., 2006, 45(1), 42-72.

[76] Sui, Y.H.; Luo, W.J.; Xu, Q.Y.; Hua, J. Dietary saturated fatty acid and polyunsaturated fatty acid oppositely affect hepatic NOD-like receptor protein 3 inflammasome through regulating nuclear factor-kappa B activation. World J. Gastroenterol., 2016, 22(8), 2533-44.

[77] Wang, Y.; Botolin, D.; Xu, J.; Christian, B.; Mitchell, E.; Jayaprakasam, B.; Nair, M.; Peters, J.M.; Busik, J.; Olson, L.K.; Jump, D.B. Regulation of hepatic fatty acid elongase and desaturase expression in diabetes and obesity. J. Lipid Res., 2006, 47, $2028-2041$.

[78] Kurek, K.; Piotrowska, D.M.; Wiesiołek-Kurek, P.; Lukaszuk, B.; Chabowski, A.; Górski, J.; et al. Inhibition of ceramide de novo synthesis reduces liver lipid accumulation in rats with nonalcoholic fatty liver disease. Liver Int., 2014, 34, 1074-1083.

[79] Geoffroy, K.; Wiernsperger, N.; Lagarde, M.; El Bawab, S. Bimodal effect of advanced glycation end products on mesangial cell proliferation is mediated by neutral ceramidase regulation and endogenous sphingolipids. J. Biol. Chem., 2004, 279(33), 34343-52.

[80] Nigro, D.; Menotti, F.; Cento, A.; Chiazza, F.; Dal Bello, F.; Romaniello, F.; Medana, C.; Collino, M.; Aragno, M.; Mastrocola, R. Chronic administration of saturated fats and fructose differently affect SREBP activity resulting in different modulation of Nrf2 and Nlrp3 inflammasome pathways in mice liver. J. Nutr. Biochem., 2017, 42, 160-71. doi: 10.1016/j.jnutbio.2017.01.010.

[81] Abe, J.; Berk, B.C. Atheroprone flow activation of the sterol regulatory element binding protein 2 and nod-like receptor protein 3 inflammasome mediates focal atherosclerosis. Circulation, 2013, 128(6), 579-82.

[82] Li, Y.; Xu, S.; Jiang, B.; Cohen, R.A.; Zang, M. Activation of sterol regulatory element binding protein and NLRP3 inflammasome in atherosclerotic lesion development in diabetic pigs. PLoS One, 2013, 8(6), e67532.

[83] Chen, Z;; Martin, M.; Li, Z.; Shyy, J.Y. Endothelial dysfunction: the role of sterol regulatory element-binding protein-induced NOD-like receptor family pyrin domain-containing protein 3 inflammasome in atherosclerosis. Curr. Opin. Lipidol., 2014, 25(5), 339-49.

[84] McRae, S.; Iqbal, J.; Sarkar-Dutta, M.; Lane, S.; Nagaraj, A.; Ali, N.; Waris, G. The Hepatitis C Virus-induced NLRP3 Inflammasome Activates the Sterol Regulatory Element-binding Protein (SREBP) and Regulates Lipid Metabolism. J. Biol. Chem., 2016, 291(7), 3254-67.

[85] Wang, W.; Wang, C.; Ding, X.Q.; Pan, Y.; Gu, T.T.; Wang, M.X.; Liu, Y.L.; Wang, F.M.; Wang, S.J.; Kong, L.D. Quercetin and allopurinol reduce liver thioredoxin-interacting protein to alleviate inflammation and lipid accumulation in diabetic rats. Br. J. Pharmacol., 2013, 169(6), 1352-71.

[86] Tian, J.; Yang, G.; Chen, H.Y.; Hsu, D.K.; Tomilov, A.; Olson, K.A.; Dehnad. A.; Fish, S.R.; Cortopassi, G.; Zhao, B.; Liu, F.T.; Gershwin, M.E.; Török, N.J.; Jiang, J.X. Galectin-3 regulates inflammasome activation in cholestatic liver injury. FASEB J., 2016.

[87] Jeftic, I.; Jovicic, N.; Pantic, J.; Arsenijevic, N.; Lukic, M.L.; Pejnovic, N. Galectin-3 Ablation Enhances Liver Steatosis, but Attenuates Inflammation and IL-33-Dependent Fibrosis in Obesogenic Mouse Model of Nonalcoholic Steatohepatitis. Mol. Med., 2015, 21, 453-65.

[88] Csak, T.; Pillai, A.; Ganz, M.; Lippai, D.; Petrasek, J.; Park, J.K.; Kodys, K.; Dolganiuc, A.; Kurt-Jones, E.A.; Szabo, G. Both bone marrowderived and non-bone marrow-derived cells contribute to AIM2 and NLRP3 inflammasome activation in a MyD88-dependent manner in dietary steatohepatitis. Liver Int., 2014, 34(9), 1402-13.

[89] Nakamura, A.; Shikata, K.; Hiramatsu, M.; Nakatou, T.; Kitamura, T.; Wada, J.; Itoshima, T.; Makino, H. Serum interleukin-18 levels are associated with nephropathy and atherosclerosis in Japanese patients with type 2 diabetes. Diabetes Care, 2005, 28(12), 2890-5.

[90] Shahzad, K.; Bock, F.; Dong, W.; Wang, H.; Kopf, S.; Kohli, S.; Al-Dabet, M.M.; Ranjan, S.; Wolter, J.; Wacker, C.; Biemann, R.; Stoyanov, S.; Reymann, K.; Söderkvist, P.; Groß, O.; Schwenger, V.; Pahernik, S.; Nawroth, P.P.; Gröne, H.J.; Madhusudhan, T.; Isermann, B. Nlrp3inflammasome activation in non-myeloid-derived cells aggravates diabetic nephropathy. Kidney Int., 2015, 87(1), 74-84.

[91] Turner, C.M.; Arulkumaran, N.; Singer, M.; Unwin, R.J.; Tam, F.W. Is the inflammasome a potential therapeutic target in renal disease? BMC Nephrol., 2014, 15, 21.

[92] Kurella, M.; Lo, J.C.; Chertow, G.M. Metabolic syndrome and the risk for chronic kidney disease among nondiabetic adults. J. Am. Soc. Nephrol., 2005, 16(7), 2134-40.

[93] Jiang, T.; Wang, Z.; Proctor, G.; Moskowitz, S.; Liebman, S.E.; Rogers, T.; Lucia, M,S,; Li, J.; Levi, M. Diet-induced obesity in C57BL/6J mice causes increased renal lipid accumulation and glomerulosclerosis via a sterol regulatory element-binding protein-1c-dependent pathway. J. Biol. Chem., 2005, 280(37), 32317-25.

[94] Iacobini, C.; Menini, S.; Ricci, C.; Scipioni, A.; Sansoni, V.; Mazzitelli, G.; Cordone, S.; Pesce, C.; Pugliese, F.; Pricci, F.; Pugliese, G. Advanced lipoxidation end-products mediate lipid-induced glomerular injury: role of receptor-mediated mechanisms. J. Pathol., 2009, 218(3), 360-9.

[95] Boini, K.M.; Xia, M.; Koka, S.; Gehr, T.W.; Li, P.L. Instigation of NLRP3 inflammasome activation and glomerular injury in mice on the high fat diet: role of acid sphingomyelinase gene. Oncotarget, 2016, 7(14), 19031-44. 
[96] Bakker, P.J.; Butter, L.M.; Kors, L.; Teske, G.J.; Aten, J.; Sutterwala, F.S.; Florquin, S.; Leemans, J.C. Nlrp3 is a key modulator of dietinduced nephropathy and renal cholesterol accumulation. Kidney Int., 2014, 85(5), 1112-22.

[97] Zoppini, G.; Targher, G.; Chonchol, M.; Ortalda, V.; Abaterusso, C.; Pichiri, I.; Negri, C.; Bonora, E. Serum uric acid levels and incident chronic kidney disease in patients with type 2 diabetes and preserved kidney function. Diabetes Care, 2012, 35(1), 99-104.

[98] Rabadi, M.M.; Kuo, M.C.; Ghaly, T.; Rabadi, S.M.; Weber, M.; Goligorsky, M.S.; Ratliff, B.B. Interaction between uric acid and HMGB1 translocation and release from endothelial cells. Am. J. Physiol. Renal. Physiol., 2012, 302(6), F730-41.

[99] Kim, S.M.; Lee, S.H.; Kim, Y.G.; Kim, S.Y.; Seo, J.W.; Choi, Y.W.; Kim, D.J.; Jeong, K.H.; Lee, T.W.; Ihm, C.G.; Won, K.Y.; Moon, J.Y. Hyperuricemia-induced NLRP3 activation of macrophages contributes to the progression of diabetic nephropathy. Am. J. Physiol. Renal. Physiol., 2015, 308(9), F993-F1003.

[100] Coughlan, M.T.; Thorburn, D.R.; Penfold, S.A.; Laskowski, A.; Harcourt, B.E.; Sourris, K.C.; Tan, A.L.; Fukami, K.; Thallas-Bonke, V.; Nawroth, P.P.; Brownlee, M.; Bierhaus, A.; Cooper, M.E.; Forbes, J.M. RAGE-induced cytosolic ROS promote mitochondrial superoxide generation in diabetes. J. Am. Soc. Nephrol., 2009, 20(4), 742-52.

[101] Gao, P.; He, F.F.; Tang, H.; Lei, C.T.; Chen, S.; Meng, X.F.; Su, H.; Zhang, C. NADPH oxidase-induced NALP3 inflammasome activation is driven by thioredoxin-interacting protein which contributes to podocyte injury in hyperglycemia. J. Diabetes Res., 2015, $2015,504761$.

[102] Ward, J.R.; West, P.W.; Ariaans, M.P.; Parker, L.C.; Francis, S.E.; Crossman, D.C.; Sabroe, I.; Wilson, H.L. Temporal interleukin-1beta secretion from primary human peripheral blood monocytes by P2X7-independent and P2X7-dependent mechanisms. J. Biol. Chem., 2010, 285(30), 23147-58.

[103] Solini, A.; Menini, S.; Rossi, C.; Ricci, C.; Santini, E.; Blasetti Fantauzzi, C.; Iacobini, C.; Pugliese, G. The purinergic $2 X 7$ receptor participates in renal inflammation and injury induced by high-fat diet: possible role of NLRP3 inflammasome activation. J. Pathol., 2013, 231(3), 342-53.

[104] Tan, C.Y.; Weier, Q.; Zhang, Y.; Cox, A.J.; Kelly, D.J.; Langham, R.G. Thioredoxin-interacting protein: a potential therapeutic target for treatment of progressive fibrosis in diabetic nephropathy. Nephron., 2015, 129(2), 109-27.

[105] Marchetti, C.; Chojnacki, J.; Toldo, S.; Mezzaroma, E.; Tranchida, N.; Rose, S.W.; Federici, M.; Van Tassell, B.W.; Zhang, S.; Abbate, A. A novel pharmacologic inhibitor of the NLRP3 inflammasome limits myocardial injury after ischemia-reperfusion in the mouse. J. Cardiovasc. Pharmacol., 2014, 63(4), 316-22.

[106] Femminò, S.; Chiazza, F.; Mastrocola, R.; Tullio, T.; Penna, C.; Nigro, D.; Alloatti, G.; Cocco, M.; Garella, D.; Bertinaria, M.; Fantozzi, R.; Aragno, M.; Collino, M. Is NLRP3 inflammasome a new pharmacological target in myocardial ischemia/reperfusion injury? Vascular Pharmacology, 2015, 75, 51.

[107] Toldo, S.; Marchetti, C.; Mauro, A.G.; Chojnacki, J.; Mezzaroma, E.; Carbone, S.; Zhang, S.; Van Tassell, B.; Salloum, F.N.; Abbate, A. Inhibition of the NLRP3 inflammasome limits the inflammatory injury following myocardial ischemia-reperfusion in the mouse. Int. J. Cardiol., 2016, 209, 215-220.

[108] Lipinski, M.J. ; Frias, J.C. Molecule 16673-34-0: a new tool in our arsenal against inflammation. J. Cardiovasc. Pharmacol., 2014, 63(4), 3145.

[109] Jong, W.M.; Leemans, J.C.; Weber, N.C.; Juffermans, N.P.; Schultz, M.J.; Hollmann, M.W.; Zuurbier, C.J. Nlrp3 plays no role in acute cardiac infarction due to low cardiac expression. Int. J. Cardiol., 2014, 177(1), 41-3.

[110] van Hout, G.P.; Bosch, L.; Ellenbroek, G.H.; de Haan, J.J.; van Solinge, W.W.; Cooper, M.A.; Arslan, F.; de Jager, S.C.; Robertson, A.A.; Pasterkamp, G.; Hoefer, I.E. The selective NLRP3-inflammasome inhibitor MCC950 reduces infarct size and preserves cardiac function in a pig model of myocardial infarction. Eur. Heart J., 2016.

[111] Wree, A.; McGeough, M.D.; Peña, C.A.; Schlattjan, M.; Li, H.; Inzaugarat, M.E.; Messer, K.; Canbay, A.; Hoffman, H.M.; Feldstein, A.E. NLRP3 inflammasome activation is required for fibrosis development in NAFLD. J. Mol. Med., 2014, 92(10), 1069-82.

[112] Yang, G.; Lee, H.E.; Lee, J.Y. A pharmacological inhibitor of NLRP3 inflammasome prevents non-alcoholic fatty liver disease in a mouse model induced by high fat diet. Sci. Rep., 2016, 6, 24399.

[113] Signorelli, P.; Ghidoni, R. Resveratrol as an anticancer nutrient: molecular basis, open questions and promises. J. Nutr. Biochem., 2005, 16, 449-466.

[114] Wu, J.M.; Hsieh, T.C.; Wang, Z. Cardioprotection by resveratrol: a review of effects/targets in cultured cells and animal tissues. Am. $J$. Cardiovasc. Dis., 2011, 1, 38-47.

[115] Szkudelski, T.; Szkudelska, K. Anti-diabetic effects of resveratrol. Ann. NY Acad. Sci., 2011, 1215, 34-39.

[116] Yang, S.J.; Lim, Y. Resveratrol ameliorates hepatic metaflammation and inhibits NLRP3 inflammasome activation. Metabolism, 2014, 63(5), 693-701.

[117] Zou, X.; Yan, C.; Shi, Y.; Cao, K..; Xu, J.; Wang, X.; Chen, C.; Luo, C.; Li, Y.; Gao, J.; Pang, W.; Zhao, J.; Zhao, F.; Li, H.; Zheng, A.; Sun, W.; Long, J.; Szeto, I.M.; Zhao, Y.; Dong, Z.; Zhang, P.; Wang, J.; Lu, W.; Zhang, Y.; Liu, J.; Feng, Z. Mitochondrial dysfunction in obesity- 
associated nonalcoholic fatty liver disease: the protective effects of pomegranate with its active component punicalagin. Antioxid Redox Signal, 2014, 21(11), 1557-70. doi: 10.1089/ars.2013.5538.

[118] Martín-Sánchez, F.; Diamond, C.; Zeitler, M.; Gomez, A.I.; Baroja-Mazo, A.; Bagnall, J.; Spiller, D.; White, M.; Daniels, M.J.; Mortellaro, A.; Peñalver, M.; Paszek, P.; Steringer, J.P.; Nickel, W.; Brough, D.; Pelegrín, P. Inflammasome-dependent IL-1 $\beta$ release depends upon membrane permeabilisation. Cell Death Differ, 2016, 23(7), 1219-31. doi: 10.1038/cdd.2015.176.

[119] Tang, B.; Chen, G.X.; Liang, M.Y.; Yao, J.P.; Wu, Z.K. Ellagic acid prevents monocrotaline-induced pulmonary artery hypertension via inhibiting NLRP3 inflammasome activation in rats. Int J Cardiol, 2015, 180, 134-41. doi: 10.1016/j.ijcard.2014.11.161.

[120] Lee, H.J.; Yeon, J.E.; Ko, E.J.; Yoon, E.L.; Suh, S.J.; Kang, K.; Kim, H.R.; Kang, S.H.; Yoo, Y.J.; Je, J.; Lee, B.J.; Kim, J.H.; Seo, Y.S.; Yim, H.J.; Byun, K.S. Peroxisome proliferator-activated receptor-delta agonist ameliorated inflammasome activation in nonalcoholic fatty liver disease. World J. Gastroenterol., 2015, 21(45), 12787-99.

[121] Benetti, E.; Mastrocola, R.; Vitarelli, G.; Cutrin, J.C.; Nigro, D.; Chiazza, F.; Mayoux, E.; Collino, M.; Fantozzi, R. Empagliflozin Protects against Diet-Induced NLRP-3 Inflammasome Activation and Lipid Accumulation. J. Pharmacol. Exp. Ther., 2016, 359(1), 45-53.

[122] Ma, C.H.; Kang, L.L.; Ren, H.M.; Zhang, D.M.; Kong, L.D. Simiao pill ameliorates renal glomerular injury via increasing Sirt1 expression and suppressing NF-кB/NLRP3 inflammasome activation in high fructose-fed rats. J. Ethnopharmacol., 2015, $172,108-17$.

[123] Wang, W.; Ding, X.Q.; Gu, T.T.; Song, L.; Li, J.M.; Xue, Q.C.; Kong, L.D. Pterostilbene and allopurinol reduce fructose-induced podocyte oxidative stress and inflammation via microRNA-377. Free Radic. Biol. Med., 2015, 83, 214-26.

[124] Samra, Y.A.; Said, H.S.; Elsherbiny, N.M.; Liou, G.I.; El-Shishtawy, M.M.; Eissa, L.A. Cepharanthine and Piperine ameliorate diabetic nephropathy in rats: role of NF- $\kappa \mathrm{B}$ and NLRP3 inflammasome. Life Sci., 2016, 157, 187-99.

[125] Birnbaum, Y.; Bajaj, M.; Qian, J.; Ye, Y. Dipeptidyl peptidase-4 inhibition by Saxagliptin prevents inflammation and renal injury by targeting the Nlrp3/ASC inflammasome. BMJ Open Diabetes Res. Care, 2016, 4(1), e000227. 